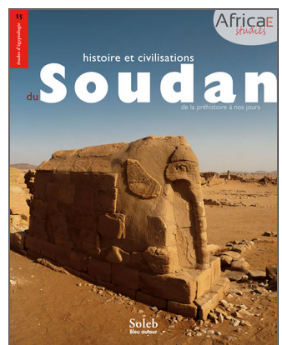

Olivier Cabon (dir.)

Histoire et civilisation du Soudan

De la préhistoire à nos jours

\title{
Rites du mariage
}

Odile Nicoloso et Nicolas Beaumé

DOI : 10.4000/books.africae. 2977

Éditeur : Africae, Soleb, Bleu autour

Lieu d'édition : Paris, Khartoum

Année d'édition : 2017

Date de mise en ligne : 17 janvier 2022

Collection : Africae Studies

EAN électronique : 9782493207074

\section{(2) OpenEdition}

\section{Books}

http://books.openedition.org

\section{Référence électronique}

NICOLOSO, Odile ; BEAUMÉ, Nicolas. Rites du mariage In : Histoire et civilisation du Soudan : De la préhistoire à nos jours [en ligne]. Paris, Khartoum : Africae, 2017 (généré le 28 janvier 2022). Disponible sur Internet : <http://books.openedition.org/africae/2977>. ISBN : 9782493207074. DOI : https:// doi.org/10.4000/books.africae.2977. 

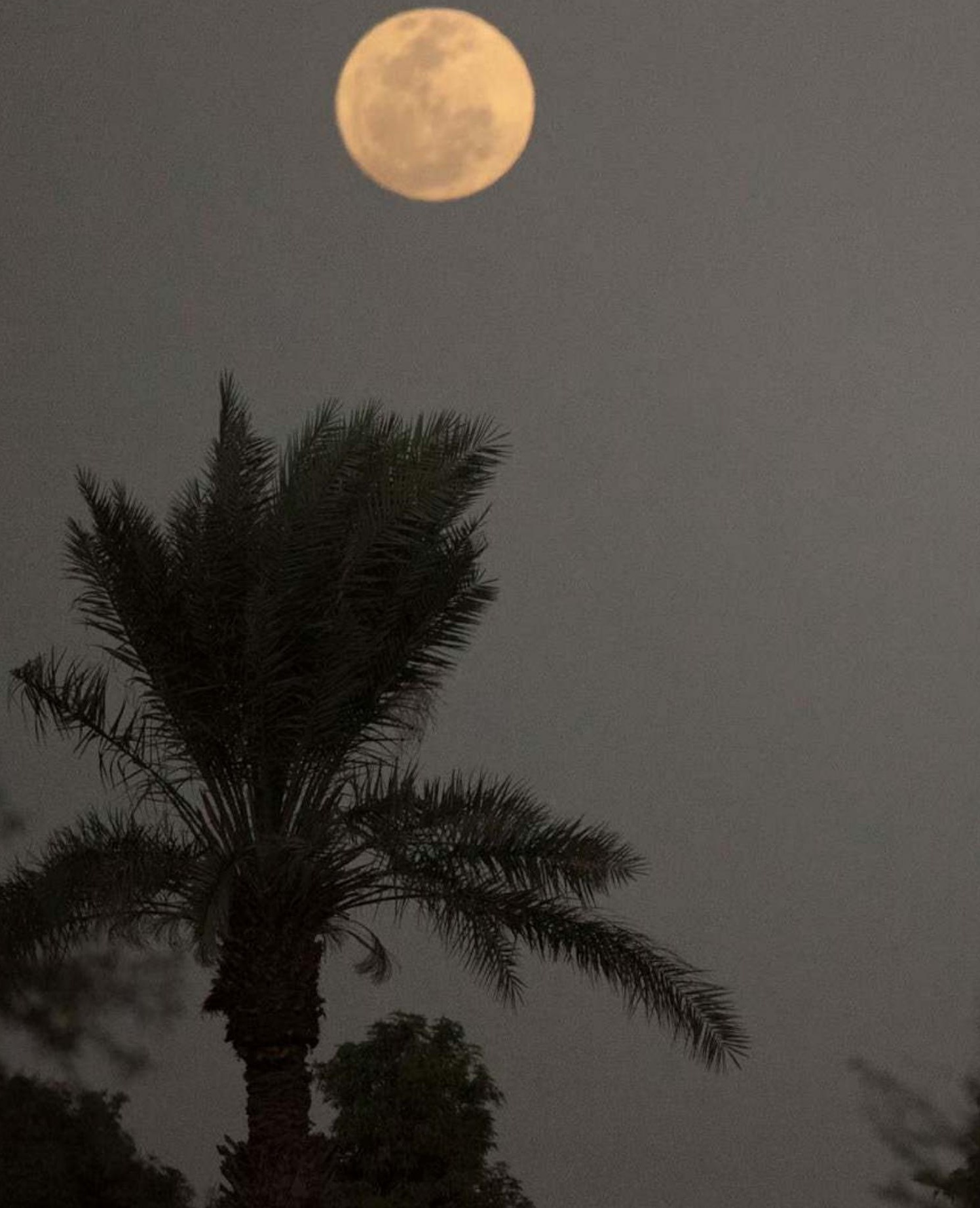

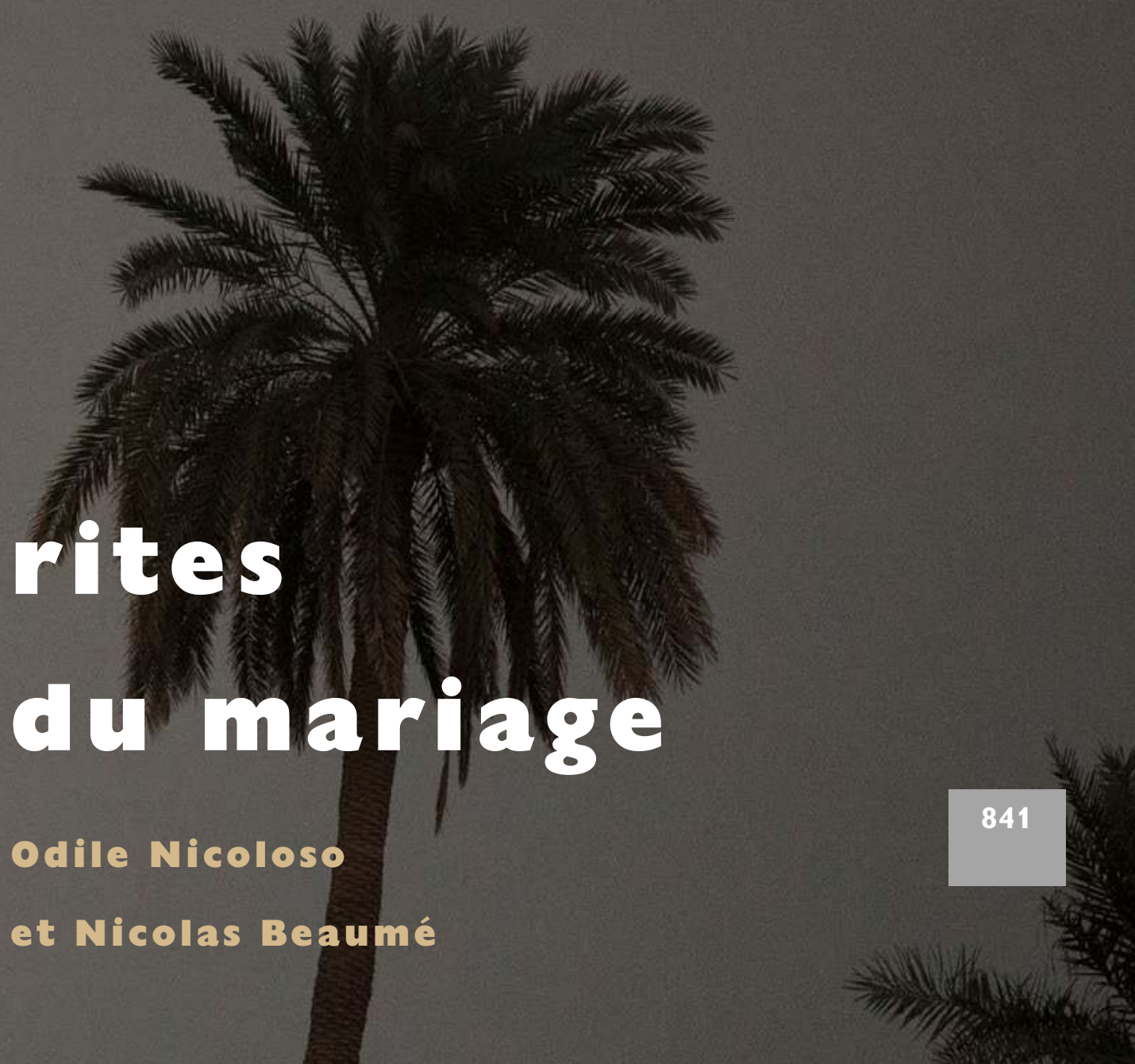
Le mariage au Soudan est une cérémonie très particulière. Avec l'évolution des mœurs, certaines coutumes ont sombré dans l'oubli mais d'autres se maintiennent dans les villages et dans les familles qui restent attachées à la tradition. C'est la grande affaire de la vie, on en parle beaucoup et très tôt. Il était et reste encore arrangé par les familles, voire au sein des mêmes familles. La cérémonie elle-même est très codée et comporte plusieurs étapes.

\section{la demande en mariage}

le Soudan

\section{2}

aujourd'hui

Autrefois le jeune homme ne pouvait en aucun cas faire sa demande luimême: une femme plus âgée s'en chargeait. S'il convoitait une jeune fille sollicitée, il la rencontrait au moins une fois avant de se déclarer. La famille de la fille avait alors deux semaines pour donner sa réponse. Ces coutumes sont tombées en désuétude, les mariages sont de moins en moins arrangés et correspondent à une vraie attirance mutuelle. Les jeunes gens peuvent désormais se rencontrer à l'université, sur leur lieu de travail, dans les cafés ou restaurants, parfois en cachette, à l'insu des familles. Ils vont, s'ils se plaisent, informer leurs parents de leur intention de se marier. Les familles vont ensuite mener leur enquête et, si rien ne vient entraver le processus, une personne d'âge mûr ou une délégation familiale, menée par le chef de famille, viendra demander la main de la jeune fille. Deux semaines plus tard, les deux mères se rendent visite, et s'il y a consentement, les pères commenceront à préparer le mariage. Ces étapes sont dénommées fatih alkhaeshum ou gulet khir.

La prochaine rencontre aura pour objet la remise de la dot (sheyla) : l'homme donnera de l'argent, des bijoux, des parures; la fiancée offrira des parfums, des vêtements locaux : (tobs, galabiah) à la famille du fiancé. Il arrive de plus en plus souvent que la fatwa établissant le mariage soit prononcée dès ce moment-là, les jeunes, ainsi mariés, pourront tôt vivre en couple, ce qui pourrait expliquer la recrudescence de divorces au bout de quelques mois.

\section{la préparation du mariage}

Autrefois, avant son mariage, la jeune fille devait rester chez elle pendant trois mois sans sortir, mais actuellement les délais diminuent: un mois, voire une semaine. Pendant cette période, il faut pratiquer le dukhān (une sorte de sauna), suivi d'un peeling pour adoucir la peau. Ce sauna se pratique grâce à un trou creusé dans un coin du jardin - mais peut-être y en a-t-il déjà un, celui qui a servi à la mère de la fiancée: on y prépare un feu 

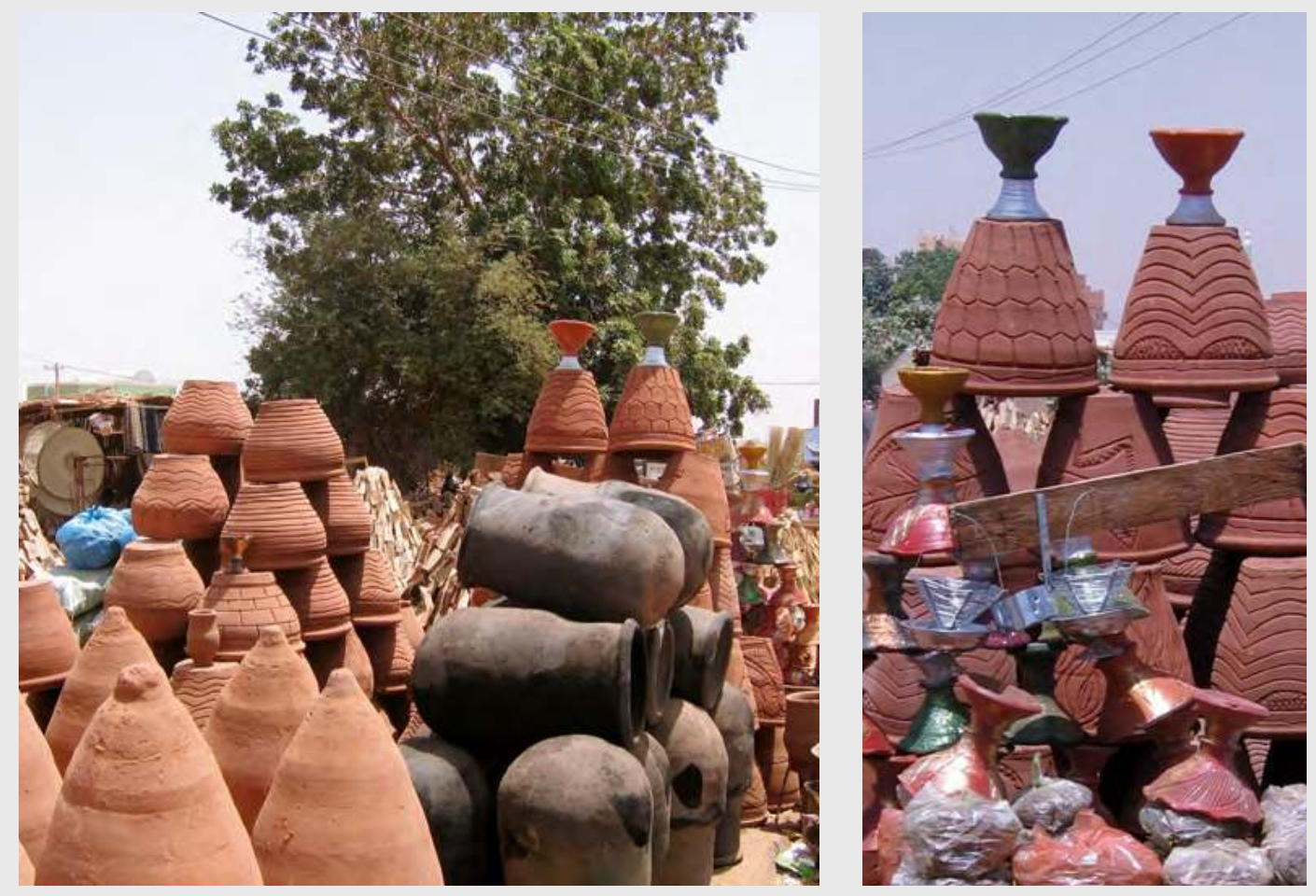

Poteries servant pour le dukhän.

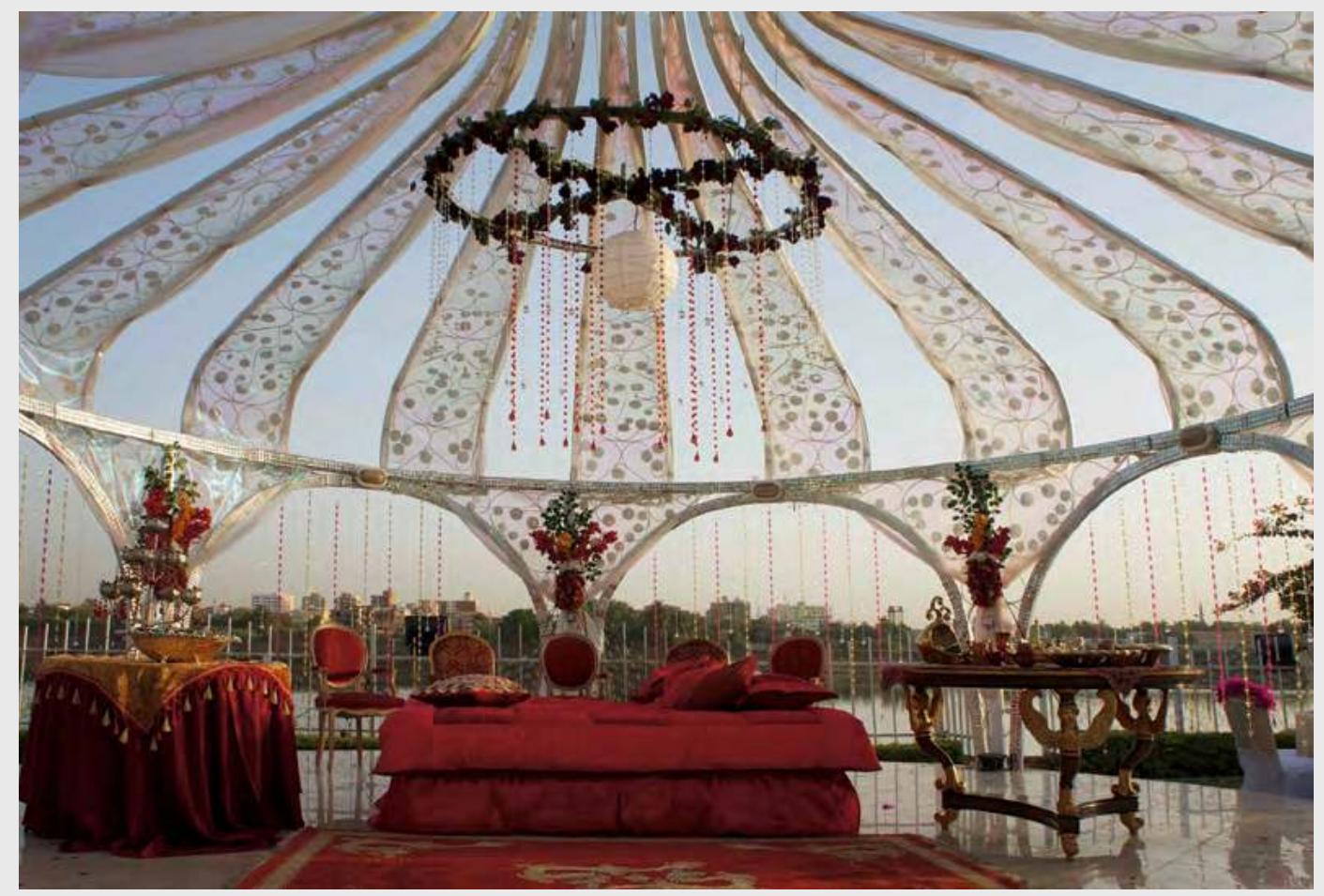




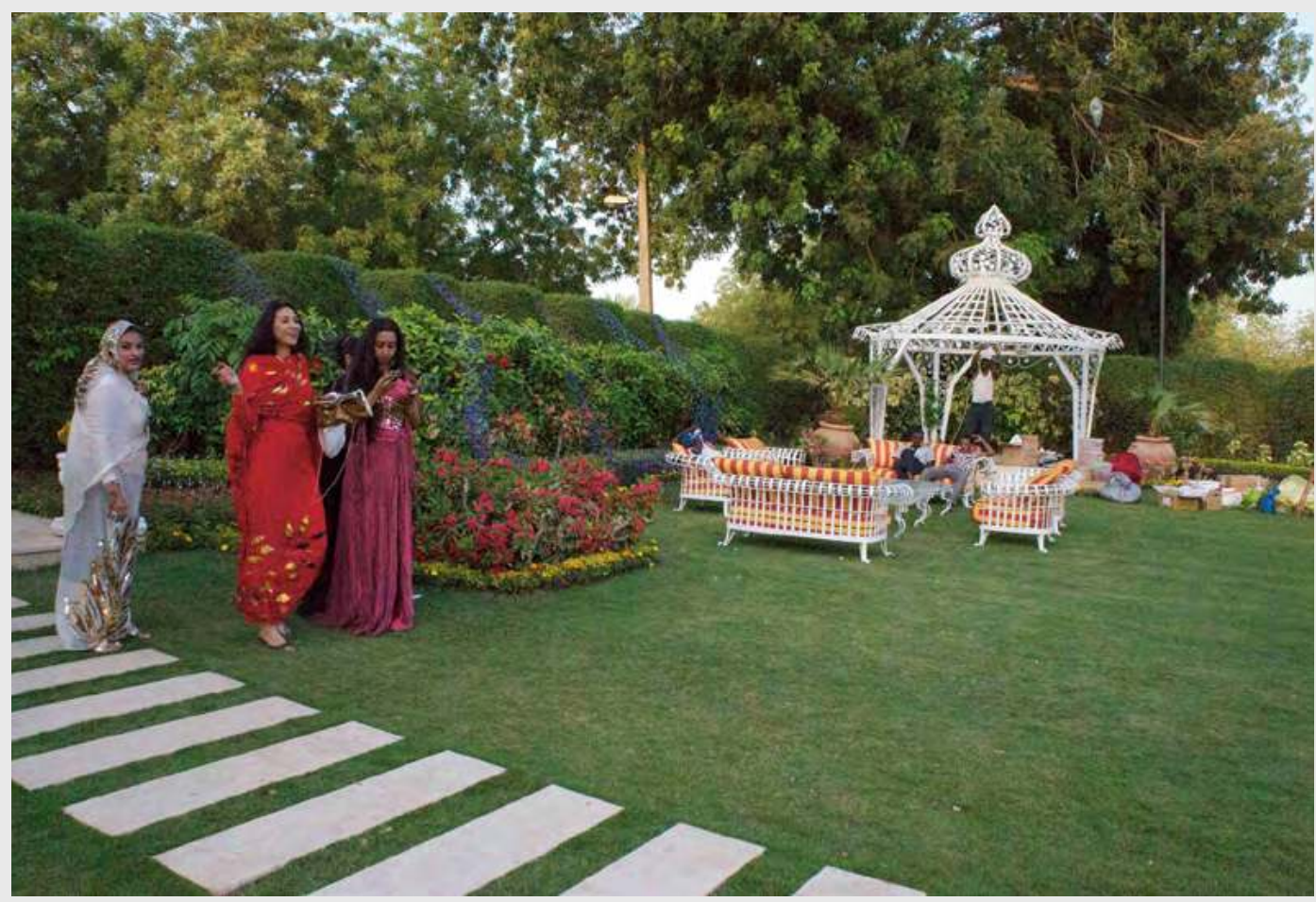

le Soudan

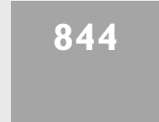

aujourd'hui

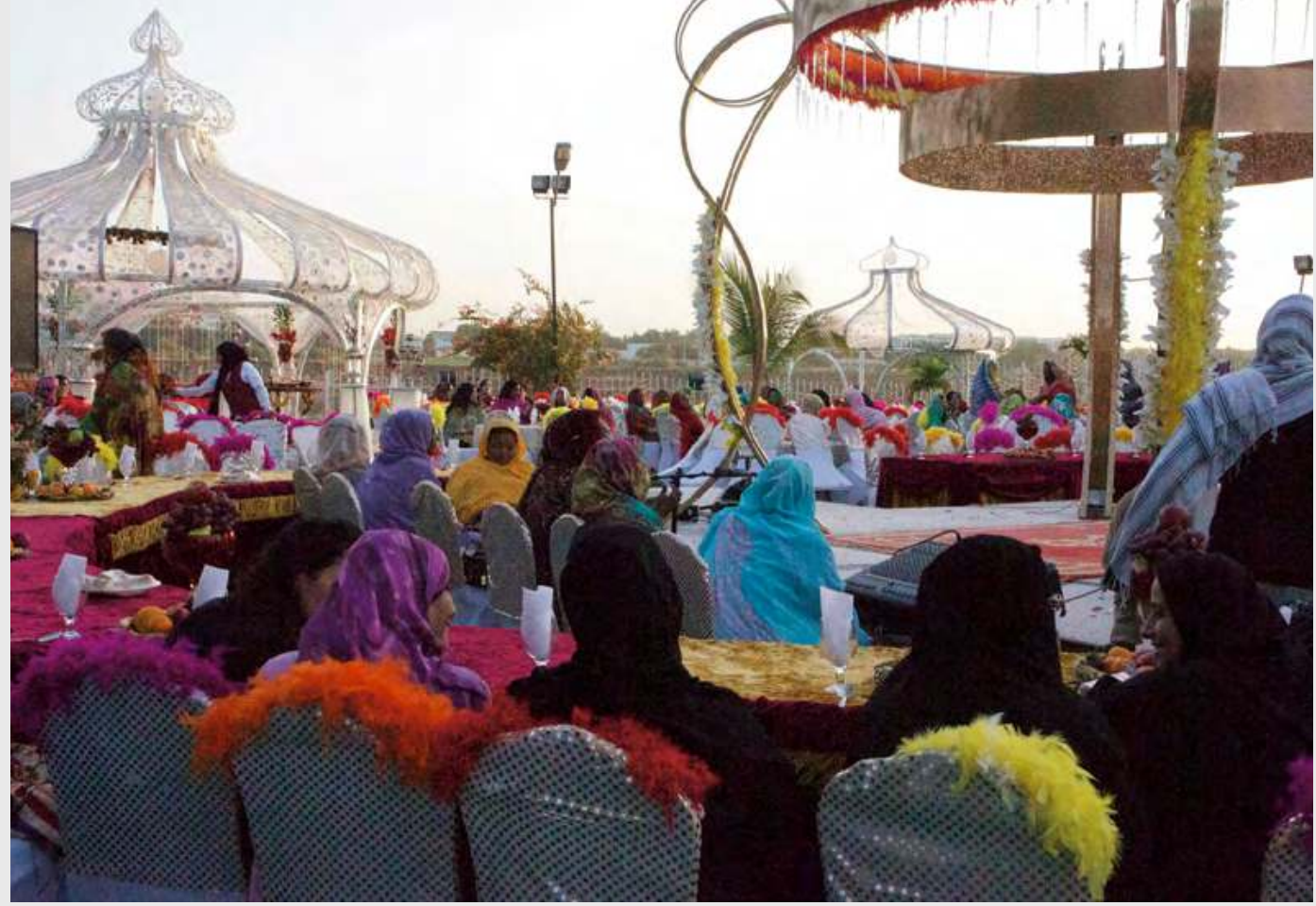

L'arrivée des invitées et le "mariage des femmes". 
de plantes odorantes; la future mariée, nue, s'assoit sur un tapis en fibres, recouverte d'une très grande couverture (shamela), ceci durant trois heures par jour. Pendant ce temps, les tantes préparent pour elle et pour ses sœurs les parfums traditionnels (santal, malhib, musk, ambre).

Ce temps de préparation au mariage est également mis à profit par la jeune fille pour apprendre à la maison, avec un professeur, les danses traditionnelles.

\section{le mariage}

Le jour précédent ou le jour même, la mariée se fait faire le henné avec ses amies. De véritables artistes dessinent sur leurs mains, leurs avants-bras, leurs pieds, leurs jambes des motifs floraux ou géométriques. Une vingtaine de ces amies apportera à la maison du futur marié henné et parfums, car lui aussi se fera faire un henné en compagnie de ses frères et amis — mais sans dessins de fleurs.

Au soir de cette journée, tous seront invités à dîner, chanter et danser. Ces fêtes du henné sont une étape importante des cérémonies: elles sont organisées à la maison, dans un club ou un hôtel.

La signature de l'acte de mariage a lieu dans la mosquée la plus proche du domicile de la mariée : c'est une cérémonie très rapide pendant laquelle le cheikh récite des versets du Coran et à laquelle assistent les proches de la famille. Elle a généralement lieu un jeudi, un vendredi ou lors d'une fête religieuse. Les mariés en sont absents. Quelques coups de feu sont tirés en l'air pour signaler au reste de la famille que le contrat a bien été signé.

Le matin du deuxième jour du mariage, la famille de la jeune fille prépare le fatouh el-arees ou "petit-déjeuner du fiancé», qu'un groupe de cinq à dix jeunes filles apportera et partagera avec famille, amis et voisins dans la maison du jeune homme.

Le soir, le marié et ses proches viennent chercher la jeune fille habillée d'une robe blanche à l'occidentale et la conduisent à la grande réception organisée pour les invités des deux familles (parfois plus de mille personnes pour les familles riches, et le nombre des invités peut être aussi très important pour des familles plus modestes).

Cette réception a lieu dans un club ou un hôtel ou sous une grande tente dressée à proximité du domicile de la mariée. Les femmes portent leurs tobs les plus colorés et les plus élégants, un orchestre et un chanteur distraient les invités. Normalement, les festivités se terminent à minuit mais parfois la fête se prolonge plus tard au domicile de l'un des deux époux. 


\section{la danse du mariage}

Enfin, le dernier soir, celui du troisième jour, a lieu la danse du mariage (sobehya), pratiquée surtout par les Arabes soudanais. La mariée dansera pour son mari et une assemblée exclusivement féminine de parentes et amies. Au cours de quatre danses différentes pour lesquelles elle changera plusieurs fois de tenue, elle chantera éventuellement, pour taquiner les autres filles célibataires et son mari.

Jusqu'au début du vingtième siècle, la mariée se ceignait les reins d'un simple rahab (ceinture d'où pendent des lanières de cuir), afin de montrer son corps et sa fertilité potentielle. Cette coutume n'est plus observée aussi strictement de nos jours: la jeune fille est un peu plus habillée, mais ses tenues successives de couleurs différentes (jaune, rouge...) restent très suggestives et elle est assez dévêtue. Des femmes policières sont souvent présentes pour empêcher la prise de toute photographie et, bien sûr, leur diffusion sur internet.

À l'issue de cette soirée - ou le quatrième jour —, a lieu le «dietek» (jirtig, jiltik), cérémonie traditionnelle à laquelle assistent seulement les proches. Sur un lit soudanais se tient la mariée en tob rouge avec, à sa gauche, le marié en galabieh blanche, le front ceint d'un bandeau orné d'un croissant. Ils échangent le sucre et le lait: celui qui le crache le premier au visage de l'autre est considéré comme le futur chef de la maison. Les femmes de la famille apportent alors sur un plateau les colliers traditionnels qui appartiennent à la famille et encensent et parfument le jeune couple pour empêcher le mauvais oil. La cérémonie se terminera par des chansons anciennes.

Le voyage de noce a lieu à l'étranger ou, pour les moins aisés, dans un hôtel de la capitale. Beaucoup de Soudanais aiment passer leur lune de miel en Érythrée. L'épouse ira ensuite généralement vivre dans la famille de son mari. Les week-ends, le jeune couple rend visite à la famille de la femme ou à d'autres parents.

Les festivités du mariage et la dot coûtent très cher. La réception durant plusieurs jours de la parentèle éloignée aussi. Faute de moyens pour financer ces dépenses, certains sont donc contraints de rester célibataires 


\section{rites

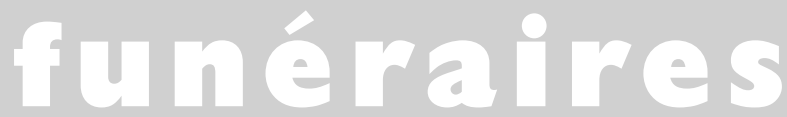

Comme ceux du mariage, les rites funéraires sont soigneusement codifiés. La personne décédée est enterrée le jour même du décès, puis la famille, les amis et les voisins ont trois jours pour présenter leurs condoléances. Les grandes familles, qui reçoivent les condoléances de centaines de personnes, installent devant la maison du défunt une grande tente destinée à l'accueil des hommes. En arrivant, le visiteur, s'il est musulman, récite la Fatiha devant le chef de famille, présente ses condoléances aux membres de la famille puis s'assoit, papote avec ses voisins en buvant du thé: dix minutes ou une heure, l'essentiel est d'être venu. La veuve, de son côté, reste chez elle vêtue de blanc pendant quatre mois et dix jours et doit, pendant cette période, se consacrer uniquement à sa peine et aux prières pour son défunt mari - 